\title{
Philosophiques
}

\section{Quelle est la norme de la perception ?}

\section{Maxime Doyon}

Volume 45, numéro 1, printemps 2018

URI : https://id.erudit.org/iderudit/1048627ar

DOI : https://doi.org/10.7202/1048627ar

Aller au sommaire du numéro

Éditeur(s)

Société de philosophie du Québec

ISSN

0316-2923 (imprimé)

1492-1391 (numérique)

Découvrir la revue

Citer ce document

Doyon, M. (2018). Quelle est la norme de la perception ? Philosophiques, 45(1),

271-278. https://doi.org/10.7202/1048627ar

Ce document est protégé par la loi sur le droit d'auteur. L'utilisation des services d'Érudit (y compris la reproduction) est assujettie à sa politique d'utilisation que vous pouvez consulter en ligne.

https://apropos.erudit.org/fr/usagers/politique-dutilisation/ 


\title{
Quelle est la norme de la perception?
}

\author{
MAXIME DOYON \\ Département de philosophie \\ Université de Montréal
}

Dans L'adresse du réel (20I7; dorénavant AR), Jocelyn Benoist aiguise la conception réaliste de la perception qu'il défend depuis au moins Éléments de philosophie réaliste (20II; dorénavant EPR) et Le bruit du sensible (2013; dorénavant BS) en mettant de nouveau de l'avant l'importante distinction entre réalité et intentionnalité, qui serait à l'origine d'un certain nombre de confusions en philosophie de la perception. Il ne faut pas confondre la chose même et sa représentation telle ou telle. "Philosophiquement, il faut clairement distinguer entre ce qui relève de la représentation et ce qui relève de la chose, entre intentionnalité et réalité. C'est là la différence ontologique fondamentale, au sens précis d'une différence entre l'être et le logos, entre ce qui est et la façon que nous avons de le déterminer en en parlant et en y pensant » (AR 280). Fidèle à la tradition oxonienne à laquelle il adhère de plus en plus, et contre une certaine tradition phénoménologique de laquelle il paraît par moment s'éloigner, Benoist nous rappelle à très juste titre la préséance de la première sur la seconde: «La grammaire de la représentation présuppose celle de la réalité, et non l'inverse » (AR 279s.).

\section{L'objet de la perception}

Dans ce contexte, l'une des questions qui se posent concerne la diversité des façons de s'orienter dans le réel, y compris les manières de déterminer cette «réalité » comme telle ou telle. Le réel est ce qu'il est, certes; c'est lui que je vois dès lors que j'ouvre les yeux. Mais le réel en tant qu'il est perçu comme tel signale qu'il est élevé au rang d'objet, c'est-à-dire de norme par rapport à laquelle il est identifié comme étant cet être et non tel autre. "Dire que la perception est "perception d'un objet" signifie placer le perçu sous une norme: celle d'une identité qui lui est attribuée. Pas d'objet sans possibilité de récognition. Ce n'est pas une propriété de l'objet. C'est sa définition» (AR 27I ; cf. BS 53). Si le réalisme de Benoist cherche à fixer et maintenir l'écart entre le factuel et le normatif, le passage d'une catégorie à l'autre joue un grand rôle dans ses analyses puisque c'est précisément là qu'il situe la possibilité de déterminer le réel, et donc, de lui conférer un sens.

Le sensible joue un rôle central dans ce travail d'objectivation du réel. Non seulement perçoit-on le réel en se familiarisant avec certains de ses aspects sensibles, mais ceux-ci sont également constitutifs de l'identité du perçu. Non pas que de tels aspects suffisent à lui conférer son identité, bien sûr; pour qu'il y ait objet, il faudra aussi "thématiser» ces aspects "afin qu'ils fassent norme » (AR 282). La normativité inhérente au concept d'objet 
suppose un écart avec le factuel que seule l'application d'une «fonction d'identité sur le réel» (AR I 22) peut venir combler. En thématisant certains de ces aspects, le réel est normé, c'est-à-dire qu'il est institué en "pôle d'identité» (AR 277). Le point est important, car c'est seulement en tant qu'il "peut être reconnu comme "le même" » (AR 27I) que le réel devient «objet" et fait figure de norme. Ce qu'on appelle objet n'est à vrai dire « rien d'autre que le concept d'une telle identité » (AR 27I) à laquelle Benoist rattache la possibilité d'être nommée comme telle dans des énoncés. "C'est une partie, ou une face du concept de perception que celle-ci puisse être exprimée dans de tels énoncés» (AR 27I).

L'idée n'est pas nouvelle. Benoist soutenait déjà dans Le bruit du sensible que l'identification du perçu comme tel «I) est toujours liée au contexte dans lequel cela a un sens de dire que telle ou telle chose est perçue; 2) précisément exprimée par la façon dont il y aurait lieu de dire ce qui est perçu, dans ce cas-là » (BS 62). La thèse, qui est reprise et prolongée dans L'adresse $d u$ réel, amène Benoist à soutenir de façon très conséquente qu' " attribuer à la perception un véritable objet signifie précisément faire un pas dans l'espace "conceptuel" " (AR 273). Cette idée, qui représente un aspect important (mais non substantiel) de la thèse de John McDowell (voir AR 265-27I), vaudrait d'ailleurs aussi pour «la phénoménologie» dans la mesure où elle opèrerait avec un concept similaire de perception: en disant "que "la perception est toujours perception de quelque chose" ", la phénoménologie ne ferait que "déployer la nature proprement conceptuelle de ce qu'on appelle “perception” " (AR 272), c'est-à-dire qu'elle ne ferait rien d'autre que réitérer l'idée suivant laquelle l'objet de la perception est normé en tant qu'il est réitérable comme tel dans le logos.

Cette affirmation n'est pas fausse. Elle est même très éclairante. En même temps, elle soulève quelques questions, car la phénoménologie ne s'est manifestement pas limitée à étaler cet aspect de la perception (ce qui ne veut pas dire qu'elle ne l'a jamais fait). Une certaine phénoménologie s'est même édifiée précisément à partir de sa tentative de vouloir transformer, réformer ou à tout le moins élargir ce concept d'objet. C'est la raison pour laquelle je sourcille un peu lorsque je lis que l'objet que l'on qualifie comme étant «le même" est celui qui, "en tant qu'objet, peut être l'objet d'autres attitudes mentales" (AR 273; je souligne), alors qu'on sait très bien que la phénoménologie (et aussi le pragmatisme américain) a tôt fait de reconnaître en outre une identité que je qualifierais pour faire court de "pratique » à l'objet de la perception, affirmant même à l'occasion la priorité de cette dernière sur la première. Si cette notion d' «identité pratique» est certes moins évidente à repérer chez le jeune Husserl, il me semble difficilement contestable qu'elle soit puissamment à l'œuvre chez Heidegger, Merleau-Ponty et tous les phénoménologues contemporains rattachés au mouvement «énactif», ce que Jocelyn Benoist n'est évidemment pas sans savoir. 
Ainsi, la question se pose de savoir s'il est possible, ou philosophiquement fécond, de rattacher le concept d'objet uniquement aux attitudes mentales que l'on peut avoir à son égard - ce que les derniers livres de Benoist donnent souvent à penser, même s'il ne l'affirme jamais explicitement. C'est que nos formes d'engagement avec le réel sont multiples et, pour une majorité d'entre elles, la question du logos de la chose ne se pose tout simplement pas. L'objet de la perception n'est normalement pas l'objet que je nomme, ni même celui dont je parle (même si je peux faire tout cela aussi); l'objet de la perception est d'abord et avant tout celui avec lequel j'interagis ou transige (transact), pour reprendre l'expression de Dewey. L'identité de l'objet se joue aussi à ce niveau — du moins pour certains objets et dans certains contextes. Ne pourrait-on pas alors dire que l'identité de la chose peut aussi se cristalliser à travers la quantité indéfinie (mais non infinie) de possibilités d'interaction qu'elle autorise ou rend possible? Considérons cette chaise, sur laquelle je suis assis. C'est en tant qu'elle me fournit à chaque fois l'occasion de m'asseoir qu'elle est ce qu'elle est (pour moi). Ce n'est pas en tant qu'être doué de parole et de langage que je lui confère, chaque fois, son identité de chaise; dans le contexte dans lequel elle figure le plus souvent, cette chaise est d'abord et avant tout ce qui m'offre chaque fois la possibilité de m'asseoir. C'est en tant qu'elle assure cette fonction (pratique) qu'elle est cette chose et non telle autre, c'est-à-dire un "objet».

L'un des héritages importants de la phénoménologie post-husserlienne réside, à mon sens, justement dans cette puissante tentative de penser de façon cohérente la question de l'identité de l'objet de la perception à partir des notions de conscience incarnée et d'intentionnalité motrice, qu'on trouve en germes chez Husserl dans le cours de 1907, Chose et espace, avant d'être développées de façon plus systématique dans la Phénoménologie de la perception de Merleau-Ponty. L'une des leçons à tirer de ces analyses consiste à reconnaitre que la caractérisation traditionnelle d'objet en tant qu'identité réitérable dans le logos n'épuise pas la notion d'objet (ce qui ne veut pas dire qu'elle ne convient pas aussi). De la même façon qu'il est vrai que tel ou tel aspect sensible ouvre la possibilité qu'on parle de l'objet d'une certaine manière, il est également vrai que ce même aspect peut aussi permettre, dans d'autres occasions, certaines possibilités d'actions ou d'interactions par le biais desquelles son identité d’objet sera révélée ou confirmée. Dans certains contextes, la priorité de cette "dimension pratique» de notre rapport à l'objet paraît difficilement contestable, comme c'est le cas par exemple pour l'improvisateur de jazz qui entend tel ou tel segment comme étant propice à son intervention ou encore comme un signe qui marque la reprise du thème ou de la mélodie. Bref, la perception ouvre non seulement la possibilité de thématiser l'être comme tel ou tel, mais elle ouvre aussi un champ d'actions possibles, et c'est souvent à l'aune de la pertinence (contextuelle, situationnelle) des actions qui sont à effectuer (ou qui pourraient l'être) que le perçu acquiert son identité, et partant, son sens. 
Si, donc, je ne suis pas en désaccord avec la caractérisation de Benoist quant aux liens à faire entre l'identité de la chose et ce que nous pourrions vouloir en dire, ses analyses ne semblent pas tenir compte de la variété d'occasions et de façons que nous avons de nous rapporter aux objets de la perception. Un privilège est accordé à quelques-uns de ces rapports au détriment d'autres, mais il n'est pas évident que cette stratégie soit pleinement justifiée. Elle n'est peut-être même pas délibérée. Voilà donc un aspect du problème qui mérite d'être précisé — ne serait-ce que pour rejeter la possibilité que je viens d'esquisser.

La question me semble d'autant plus importante qu'elle a une incidence directe sur la conception du normatif qui est mise de l'avant tout au long de l'ouvrage. En effet, si le concept d'identité pratique n'est pas tout à fait irrecevable, alors le concept de «norme de la perception » demanderait à être élargi en conséquence. Schématiquement: si, en tant que pôle d'identité, l'objet se laisse appréhender comme tel tout autant par les actions qu'il rend possible que par les énoncés et les pensées qu'il permet, alors l'objet de la perception serait normatif dans un autre sens que celui envisagé par Benoist. Il peut être plus ou moins bien vu ou entendu, par exemple, m'incitant à modifier ma perspective sur lui. Une question très générale se pose donc ici: nos prises normatives sur le réel relèvent-elles toutes du langage, ou y a-t-il des normes propres à la perception elle-même, c'est-à-dire avant ou à tout le moins indépendamment de son éventuelle mise en langage? Même si l'on s'accorde avec Benoist sur le fait que «le langage est le paradigme de toute norme» (AR I 20), peut-être faudrait-il tout de même faire une place dans nos analyses pour une autre forme de normativité. Je ne pense pas que cette proposition aille nécessairement à l'encontre des thèses avancées par Benoist dans L'adresse du réel, mais elle pointe vers un élargissement possible de l'analyse qu'il nous propose ${ }^{1}$.

\section{Illusions et hallucinations}

L'une des conséquences de la réflexion précédente sur la normativité de la perception touche la conception de l'illusion et l'hallucination que Benoist élabore en faisant travailler une autre notion d'écart. Benoist se demande «par rapport à quoi [...] on prétend formuler un écart » (AR 245) lorsque nous sommes victimes d'une illusion ou d'une hallucination. Comment qualifier cet écart? De quel type d'écart s'agit-il?

Dans L'adresse du réel, Benoist se penche sur cette série de questions à partir d'une Auseinandersetzung avec la théorie disjonctive, celle de Mike Martin en particulier, au sein de laquelle ledit écart est entendu en termes

1. C'est ce que j'ai tenté de faire dans plusieurs articles récents, dont «Perception and Normative Self-Consciousness ", qui fait partie du collectif Normativity in Perception, T. Breyer et M. Doyon (dir.). 
exclusivement épistémiques². L'une des ambitions claires de Benoist est de démontrer la non-pertinence de cette caractérisation et, plus généralement, de l'ensemble des catégories épistémiques traditionnellement admises en philosophie de la perception (comme la notion de facteur commun, l'opposition entre l'internalisme et l'externalisme, ou encore celle entre le disjonctivisme et le conjonctivisme, etc.), qui semblent toutes « reposer largement sur une pure construction philosophique»(BS 8I), dépeignant ainsi un «faux débat» (BS 75) sur la nature de la perception. Benoist a tout à fait raison d'y insister: il y a quelque chose d'artificiel et de hautement "spéculatif» (BS 79) à analyser illusions et hallucinations dans les limites d'un tel cadre. Les illusions ne sont pas des perceptions fausses, ni les hallucinations de fausses perceptions. Sauf circonstances particulières, il ne s'agit pas là de concepts pertinents pour caractériser ces expériences. Les illusions ne sont ni vraies ni fausses, elles sont réelles, et cela veut dire qu'elles comportent, irréductiblement, une dimension sensible. Ainsi, l'un des objectifs poursuivis par Benoist consiste à fournir une «analyse de la perception en tant que réalité » (BS 8I).

En tant qu'elles sont réelles, les «illusions» sont de plusieurs genres. Il y a, par exemple, les illusions d'optique, qui sont artificiellement construites pour nous tromper ou nous méprendre. C'est notamment le cas de l'illusion de Müller-Lyer, dans lequel le jugement perceptif est en décalage avec la chose même. Mais de quel type d'erreur s'agit-il ? La thèse défendue par Benoist ici (qui est aussi celle de Travis et d'Austin) est que l'erreur est judicative, et non perceptive. Précisément, l'erreur vient du fait que, parfois, et "compte tenu des contraintes de notre vie pratique et de nos routines perceptuelles ", nous sommes "plus ou moins à l'aise » avec tels ou tels aspects (AR 234) de la scène perçue. Les illusions d'optiques sont «des perceptions avec lesquelles nous avons des problèmes » (AR 235). Or, puisque ces problèmes peuvent être variés et de différents types, qualifier en bloc toutes ces perceptions de "perceptions fausses» traduit, selon Benoist, une certaine pauvreté analytique, pauvreté qui ferait elle-même système avec une profonde cécité phénoménologique: "traiter les "illusions" comme des "perceptions fausses", c'est vouloir ignorer la variété de la teneur de ce que nous appelons “"perception” » (AR 233). Encore une fois, difficile d'être en désaccord avec Benoist sur ce point.

Dans le cas des illusions «réelles» ou «mondaines» (comme par exemple celle du «bâton dans l'eau»), Benoist insiste - encore une fois à très juste titre - pour dire que ce sont là essentiellement des perceptions comme les autres. Il n'y a en effet rien d'anormal ou de faux dans ma perception lorsque je prends un bâton droit qui est dans l'eau pour un bâton

2. L'analyse du chapitre VII relaie, en la complétant, celles avancées dans Éléments de philosophie réaliste (chap. IV) et Le bruit du sensible (chap. III); c'est pourquoi je puiserai ici des idées et des citations dans ces trois ouvrages. 
recourbé. Tout simplement je commets une erreur de jugement qui, pour faire court, s'explique le plus souvent par une négligence du contexte de ma perception (le fait que le bâton est dans l'eau et non à l'air libre, par exemple).

Mais - et on retrouve ici un thème développé plus tôt - avons-nous ici tout dit des circonstances où une telle scène risque de se dérouler ? Car on peut faire le même point en considérant non pas nos jugements de perceptions, mais l'action sur laquelle la perception débouche. A priori, les illusions peuvent se révéler comme telles autant dans un jugement modifié ou corrigé (y compris par autrui) qu'à travers les diverses façons d'interagir avec les choses. C'est, par exemple, en manipulant (ou en cherchant à manipuler) le bâton que je me rends compte qu'il est droit et non recourbé. L' «erreur» s'est d'abord manifestée dans ma façon de (vouloir) le saisir ou dans l'usage que je peux maintenant en faire. L'exemple illustre comment le changement d'attitude eu égard à la chose qui marque la conscience de l'illusion peut très bien se jouer d'abord dans l'action avant d'être corroboré par mon jugement ou ma croyance. En d'autres termes, si les illusions sont «des perceptions avec lesquelles nous avons des problèmes " (AR 235), ces problèmes peuvent très bien se manifester dans notre façon d'interagir avec les choses. En ce sens, il me semble que l'examen du lien intentionnel (ou de la boucle de rétroaction) entre action et perception est tout aussi important que l'analyse du langage ou des concepts de la perception pour l'analyse des illusions.

Un point similaire peut être fait à partir du concept d'hallucination. L'hallucination est une épreuve de réalité, et non une prise sur la vérité. Elle est «réelle» et non «vraie». Cela veut dire au moins deux choses, selon les deux types d'hallucinations que Benoist distingue. Il y a, d'une part, les hallucinations réelles, c'est-à-dire cliniques, à propos desquelles Benoist souligne à très juste titre qu' «il est relativement peu commun qu'un agent soit épistémiquement fourvoyé par une hallucination» (BS 83) $)^{3}$. D'autre part, il y a les hallucinations perceptuelles, qu'il appelle maintenant "hallucinations visuelles de déformation» ( $\mathrm{AD}$ 24I). Si Benoist reconnaît qu' "à ce niveau [...] la ligne de partage entre "hallucination" et "illusion" n'est pas facile à tracer» $(\mathrm{AR} 239)^{4}$, il tente néanmoins le coup en comparant les hal-

3. Selon Benoist, la victime de l'hallucination est un peu comme un sujet socratique: dans une vaste majorité de cas, l'halluciné «sait bien alors qu'il ne sait pas» (BS 84). Ici, le «trouble l'emporte, un trouble qui déstabilise l'agent en tant que sujet de connaissance (BS $83)$.

4. À cet égard, Benoist démontre que la distinction ne coïncide pas avec la définition traditionnelle (d'Esquirol) de l'illusion et de l'hallucination comme perception déformante d'un objet (réel) et apparaître sans objet respectivement. L'un des problèmes de ce partage, c'est que l'idée d'un pur apparaître semble être «un cas-limite» (AR 242), car «il n'y a pas d'hallucination à l'état isolé, ou en tout cas absolument isolé: l'hallucination surgit sur un fond essentiel de réalité" (BS 86). En ce sens, elle est aussi "déformante» que l'illusion et s'en distingue d'autant plus difficilement. 
lucinations aux illusions d'optiques. Alors que l'illusion d'optique est une perception "d'un air qu'a objectivement » la chose perçue, l'hallucination a quelque chose de privé et "rompt précisément avec cet air " ${ }^{5}$. On comprend maintenant mieux le caractère réel de l'hallucination que Benoist veut faire ressortir: non seulement l'hallucination "git sur un fond essentiel de réalité » (BS 86) qu'elle déforme, mais «même ce qui est halluciné est réel »: «il est exactement ce qui est vu - ou, plus communément, entendu. Comme telle, l'hallucination est un moment réel de cette relation à la réalité qu'est notre perception - quelque chose qui peut se produire en elle» (BS 87). Il appert que l'hallucination a lieu dans le réel et donc au sein de la perception, comme un moment de celle-ci. Les hallucinations "sont une partie de ce que nous voyons, entendons, sentons » et, en ce sens précis, elles font donc intégralement partie "de la facticité du perçu » (BS 87).

En tant que telle, l'hallucination possède une phénoménalité qui lui est propre, voire unique. Elle est «comme une excroissance locale de la perception» (BS 86), c'est-à-dire qu'elle est caractérisée «par une forme de saveur excessive de la réalité " (BS 83). La même idée est reprise dans L'adresse du réel: «En effet, ce qui caractérise souvent l'expérience de type hallucinatoire, c'est paradoxalement sa très forte trempe de réalité. Ce qui apparaît dans l'hallucination apparaît avec une réalité insoutenable et est, en quelque sorte, "trop réel pour être vrai" " (AR 256). Si parler de "surréalité » dans leur cas, ou invoquer "leur extrême réalité» (AR 256) permet à Benoist d'expliquer, d'une part, pourquoi «la question de l'indistinguabilité épistémique ne se pose pas: il n'y a aucun risque que le sujet prenne épistémiquement l'hallucination pour une perception de réalité" (AR 256), elle lui permet également, d'autre part et "pour la même raison ", de rejeter la thèse de "l'indistinguabilité phénoménale», qui "est également impossible: les hallucinations, avec leur surréalité extrême — leur réalité extrême, pourraiton dire - ont une qualité phénoménale particulière que la perception n'a pas» (AR 256). Bref, Benoist semble voir dans cette caractérisation la «spécificité phénoménologique» (AR 258) des hallucinations qui lui permet de faire certaines avancées sur le plan conceptuel.

Non sans lucidité, Benoist émet quelques réserves quant à la portée de cette caractérisation lorsqu'il reconnaît que «l'argument de Gonzalez»sur lequel cette caractérisation s'appuie largement semble un peu "excessif» (AR 257). Faute d'espace, je n'étalerai pas les raisons de ses hésitations (et des miennes); je saisirai plutôt l'occasion pour réfléchir sur ce qui pourrait constituer les contours d'une phénoménologie de l'hallucination. Il va sans dire que l'élaboration d'une telle phénoménologie dépasserait très largement le cadre imposé par le genre d'exercice auquel je me prête ici, mais j'aimerais

5. Cependant, J. B. est aussi lucide quant aux limites de ce découpage: «Il n'est pas facile de savoir ce qu'il y a un sens à placer respectivement du côté de l'objet et du côté du sujet » (AR 242), ce qu'il démontre à partir d'une analyse comparative fort riche de la myopie. 
néanmoins fournir deux très brèves pistes de réflexions qui me permettront de renouer avec mon thème principal.

La première concerne le temps, ou l'aspect spécifiquement temporel de l'expérience. En moyenne, les hallucinations cliniques ont tendance à durer plus longtemps que les illusions réelles et souffrent par rapport aux perceptions. Alors que les objets perçus ont une structure horizontale qui ouvre sur des possibilités quasi infinies d'exploration, «l'hallucination glisse dans le temps ( $\mathrm{PP}$ 397) affirme très justement Merleau-Ponty, et s'estompe. Précisément en vertu de son caractère éphémère - et voici le deuxième trait que je voulais souligner - la chose hallucinatoire empêche l'agent de l'explorer ou de la manipuler durablement. La chose hallucinatoire n'a ni profondeur ni épaisseur, et donc aucune action durable n'est possible à son égard. $L a$ boucle de rétroaction entre la perception et l'action est pour ainsi dire "rompue». Le lien intentionnel est défait. Ce qui revient à dire que le sujet délirant se retrouve en déficit pratique: il ne peut pas exercer habilement (c'est-à-dire normalement) ses compétences perceptuelles et agir comme il le fait habituellement sur la base de ses perceptions.

Ces deux critères nous permettent de voir en quel sens toutes les expériences de type perceptuel ne s'équivalent pas: certaines sont mieux réussies que d'autres, c'est-à-dire plus en phase avec notre activité ou notre projet, qu'elles contribuent à réaliser ou à faire progresser, alors que d'autres commandent certains ajustements. Or d'autres encore s'avèrent tout à fait incompatibles avec l'idée même d'un tel ajustement. Alors qu'une perception est dite "réussie ", voire "optimale", si elle s'intègre bien dans mon projet intentionnel global, c'est-à-dire si elle s'inscrit dans la poursuite de mes objectifs et répond à mon intérêt $t^{6}$, l'hallucination semble exclure ou à tout le moins compliquer sérieusement toute possibilité d'optimiser notre expérience (sauf pour en sortir, pour ainsi dire).

Nous retrouvons ici notre fil conducteur: si je suis parfaitement d'accord avec la stratégie de Benoist qui souligne à grands traits l'importance du contexte de la perception, il me semble évident, en revanche, que cette sensibilité au contexte est aussi incarnée. Ainsi, je me questionne sur le sens à donner à l'absence virtuelle de toute considération du rôle du corps dans les analyses de Benoist lorsqu'il s'agit de préciser la nature de la normativité propre à l'activité perceptuelle. La question est de savoir s'il s'agit d'un choix assumé qui s'appuie sur des motivations philosophiques claires.

6. Voir sur ce point ma plus récente étude: «Husserl on Perceptual Optimality », in Husserl Studies 2018. 